\title{
Less than 1000 days to go for MDGs 4 and 5: where are we and what needs to be done?
}

\author{
Flavia Bustreo ${ }^{7}$
}

The Millennium Development Goals (MDGs) 4 and 5 called for reducing the mortality of under-5-year-olds by two-thirds between 1990 and 2015, and reducing maternal mortality by three-quarters over the same period. Substantial progress towards this has been made worldwide. The global maternal mortality ratio fell by $47 \%$ between 1990 and 2010 and the under-5 mortality rate also fell by $47 \%$ between 1990 and $2012[1,2]$. However, there has been insufficient progress and there is a risk that the global targets will not be met. Still every year 6.6 million children die before their 5th birthday, of whom 2.9 million are newborn babies in the first month of life. An estimated 287000 women die due to the complications of pregnancy and childbirth, and the annual 2.6 million stillbirths are silent tragedies that have to be prevented.

The leading causes of maternal mortality-obstetric haemorrhage, hypertensive disorders of pregnancy, sepsis and unsafe abortion - are to a large extent preventable in most highburden countries. Certain services are key to preventing maternal deaths, such as contraception, antenatal care, skilled attendance at birth and postnatal care. Likewise, many children still die from easily preventable diseases. Deaths in the newborn period are mostly due to prematurity, sepsis and intrapartum causes, while in the 1-59-month age group it is pneumonia and diarrhoea and high rates of malnutrition which underlie over $45 \%$ of all under- 5 deaths. In addition, malaria and HIV and AIDS cause significant numbers of deaths in some countries. For those children who survive, malnutrition may jeopardize their potential for optimal growth and development, with significant consequences later in life.

Proven effective interventions exist to further reduce maternal and child mortality [3], and the scaling up of these interventions is critical. However, coverage remains low for many of these interventions and is often not evenly distributed within countries. Recognizing that progress in maternal, newborn and child survival requires reaching all population subgroups with essential health services, health-equity dimensions such as wealth, sex, age, maternal education, ethnicity and urban/rural residence are critical factors to take into account. Analysis of data in the latest Countdown to 2015 report showed pronounced inequities in coverage for many essential interventions, with women and children from richer households much more likely to receive care than those from poorer households [4]. This pattern is particularly evident for interventions that require a fully functional health system, such as having a skilled attendant at the birth.

Multiple global efforts are being made to support countries to accelerate progress and address the "unfinished business" of reducing maternal and child mortality. The United Nations Secretary-General's Global Strategy for Women's and Children's Health, launched in 2010, is an unprecedented plan to save the lives of 16 million women and children by 2015 .
Under this umbrella, the Commission on Information and Accountability for Women's and Children's Health developed a framework to monitor and track commitments made to the Global Strategy, and an independent Expert Review Group (iERG) reports annually on progress towards implementation of the Commission's recommendations in 75 high-burden countries regarding reporting, oversight and accountability. More recently, a number of initiatives have been launched with the aim of accelerating progress towards MDGs 4 and 5 , and to end preventable maternal and child mortality in a generation. The Commitment to Child Survival: A Promise Renewed sets out targets for reduction of child mortality to 20 child deaths or less per 1000 live births by 2035. In support of this target, the Global Action Plan for the Prevention and Control of Pneumonia and Diarrhoea (GAPPD) provides directions for ending preventable child deaths due to pneumonia and diarrhoea through a combination of interventions, such as exclusive breastfeeding and good nutrition, hand-washing, safe drinking-water, improved cooking stoves, zinc supplements and oral rehydration solution, amoxicillin, vitamin A supplements and new vaccines. Likewise, a plan for reducing newborn deaths-Every Newborn: an Action to End Preventable Newborn deaths - is in preparation to address this increasingly important issue. Finally, the Family Planning 2020 (FP2020) initiative at the London Family Planning Summit in 2012 set an ambitious goal to provide an additional 
120 million women in the world's poorest countries with access to voluntary family planning by 2020 .

These global initiatives are important drivers for sustained action and commitment for improving maternal and child health and reducing mortality. They cannot stand alone, however. The most critical factor for success is the extent to which follow-up and action is happening at regional and country levels. The WHO Eastern Mediterranean Region (EMR) has taken important steps in this direction. Nonetheless, in the EMR, the average annual rates of reduction in maternal and child mortality between 1990 and 2010 (2\% for under-5 mortality rate and $2.6 \%$ for maternal mortality ratio) are among the lowest in the world. Some countries have made tremendous progress over the last 20 years. However, the Region is unlikely to achieve the targets of MDGs 4 and 5 by 2015 unless intensive and accelerated progress is made, especially in those countries contributing to the bulk of under-5 and maternal deaths.

It is estimated that 923000 children under 5 years of age and around 39000 women of childbearing age still die every year in the Region as a result of pregnancy-related complications. Mortality rates are particularly high in the poor, rural and underserved areas, among malnourished children and pregnant adolescents. In response to that challenge, WHO, United Nations Children's Fund (UNICEF) and the United Nations Population Fund (UNFPA), in collaboration with countries of EMR and partners, have commenced a regional initiative to accelerate progress towards MDGs 4 and 5 focusing on 10 high-burden countries (Afghanistan, Djibouti, Egypt, Iraq, Morocco, Pakistan, Somalia, South Sudan, Sudan and Yemen). A high-level meeting on "Saving the lives of mothers and children: rising to the challenge", held in Dubai, United Arab Emirates on 29 and 30 January 2013, was attended by 150 participants, including 10 ministers of health, senior officials and leading figures from 22 Member States, as well as key partners and stakeholders. Through the Dubai Declaration, countries expressed their commitment to develop and execute plans for maternal and child health; to take measurable steps to strengthen their health systems; and to mobilize domestic and international resources to establish sustainable financing mechanisms.
Encouragingly, following this highlevel meeting in Dubai, all 10 countries have developed acceleration plans for MDGs 4 and 5 with support from WHO, UNICEF, UNFPA and other partners. The plans vary from country to country, reflecting their different situations and contexts. However, the plans focus on increasing coverage of the key cost-effective maternal and child health interventions across the continuum of care while addressing inequities in maternal and child health, together with a call for more intersectoral collaboration. The time for implementation of the plans is now.

Getting closer to reaching MDGs 4 and 5 and to reaching the ambitious targets set beyond 2015 will require sustained commitment by all stakeholders. It will also require a strengthening of health systems through allocation of the necessary human and financial resources to mobilize both domestic and international funding. Importantly, however, this should not be viewed as merely an additional cost burden. The health of women and children should be seen as an investment in the future that may also lead to wider societal gains and socioeconomic development.

\section{References}

1. Trends in maternal mortality: 1990 to 2010. WHO, UNICEF, UNFPA and The World Bank estimates. Geneva, World Health Organization, 2012. (http://www.unfpa.org/webdav/site/ global/shared/documents/publications/2012/Trends_in_ maternal_mortality_A4-1.pdf, accessed 9 December 2013).

2. Levels and trends in child mortality. Report 2013. Estimates developed by the UN Inter-agency Group for Child Mortality Estimation. Geneva, United Nations Children's Fund, 2013 (http:// www.childinfo.org/files/Child_Mortality_Report_2013.pdf, accessed 9 December 2013).

3. Essential interventions, commodities and guidelines for reproductive, maternal, newborn and child health. Joint press release:
PMNCH, WHO and Aga Khan University. The Partnership for Maternal, Newborn and Child Health, 15 December 2011 [online press release] (http://www.who.int/pmnch/media/ press/2011/20111215_essential_interventions_pr/en/index. html, accessed 9 December 2013).

4. Accountability for maternal, newborn and child survival. The 2013 update. Geneva, World Health Organization/United Nations Children's Fund, 2013 (http://countdown2015mnch.org/ documents/2013Report/Countdown_2013-Update_noprofiles.pdf, accessed 9 December 2013). 